\title{
O senso de auto-eficácia e o comportamento orientado para aprendizagem em crianças com queixa de dificuldade de aprendizagem $^{1}$
}

\author{
Paula Cristina Medeiros \\ Sonia Regina Loureiro \\ Maria Beatriz Martins Linhares \\ Edna Maria Marturano \\ Universidade de São Paulo
}

\begin{abstract}
Resumo
Objetiva-se avaliar aspectos do desempenho acadêmico de crianças, relacionando indicadores da produção e do comportamento orientado para aprendizagem com indicadores de percepção do senso de auto-eficácia. Procedeu-se a avaliação por meio do Roteiro de Observação Clínica Comportamental da Criança e do Roteiro de Avaliação do Senso de Auto-Eficácia de 52 crianças, de ambos os sexos, na faixa etária de oito a 12 anos, alunos de $1^{\underline{a}}$ a $4^{\underline{a}}$ série, divididas em: 26 crianças com queixa de dificuldade de aprendizagem, encaminhadas para atendimento psicológico e 26 crianças com bom desempenho acadêmico avaliado por Teste de Desempenho Escolar. Observou-se que as crianças com queixa de dificuldade de aprendizagem, quando comparadas às crianças com bom desempenho, apresentaram diferença estatisticamente significativa; a) quanto à produção: atividades de qualidade média; b) quanto ao comportamento: baixa capacidade de organização, atenção, iniciativa, decisão, comunicação e interação espontânea; c) quanto à percepção: baixo senso de auto-eficácia.
\end{abstract}

Palavras-chave: Dificuldade de aprendizagem, Senso de auto-eficácia, Comportamento.

\begin{abstract}
Sense of self-efficacy and behavior oriented towards learning in children with complaint of learning difficulty. The aim of this work has been to assess children's academic performance, relating indicators of production, and behavior oriented towards learning, with indicators of self-efficacy sense perception. Fifty-two children, from 8 and 12 years old, from both sexes have been assessed through the Guideline for Children's Behavioral Clinical Observation and through the Guideline for the Assessment of Self-efficacy Sense. They had been attending from the $1^{\text {st }}$ to the $4^{\text {th }}$ primary school levels and were divided in: 26 children with complaint of learning difficulty, forwarded to psychological attendance and 26 children with good academic performance assessed through the School Performance Test. It has been seen that the children with complaint of learning difficulty, as compared to children with good academic performance, presented statistically significant difference in the: a) production: average quality activities; b) behavior: low organization capacity, attention, initiative, decision, communication and spontaneous interaction: c) perception: low sense of self-efficacy.
\end{abstract}

Key words: Learning difficulty, Sense of self-efficacy, Behavior.

I nseridos na perspectiva atual da Psicopatologia do Desenvolvimento, com base em extensa revisão da literatura, Rutter e Sroufe (2000) e Sameroff (2000) consideram o funcionamento comportamental e biológico como parte de um sistema geral de regulação e adaptação do desenvolvimento infantil, integrando processos individuais e contextuais em um modelo dinâmico.

Este modelo partilha das proposições da visão ecológica do desenvolvimento (Bronfenbrenner, 1994) considerando a escola, além da família, um dos primeiros contextos sociais que proporciona à criança o contato com novas oportunidades ampliando o universo de interação com adultos e crian- ças. Segundo Roeser e Eccles, (2000), as experiências escolares tanto podem promover competências associadas à aprendizagem, à motivação para a realização, ao funcionamento emocional e relacionamentos sociais, quanto podem, em algumas instâncias, potencializar as dificuldades escolares, favorecendo a instalação de um padrão desordenado de adaptação.

A psicopatologia do desenvolvimento contribui para a identificação de fatores que influenciam as habilidades das crianças para organizar experiências e, conseqüentemente, o seu nível de funcionamento adaptativo (Sameroff, 2000). Dentro deste modelo dinâmico considera-se a influência de múlti- 
plas variáveis sobre o comportamento das crianças, incluindo entre estas as autopercepções, especificamente o senso de auto-eficácia.

Bandura (1982) definiu auto-eficácia como a crença do indivíduo sobre a sua capacidade de desempenho em atividades específicas. No que diz respeito ao desempenho acadêmico o senso de auto-eficácia afeta "o quê" os estudantes fazem influenciando as escolhas de atividades, o estabelecimento de metas, o esforço despendido, a persistência e perseverança frente às adversidades e o nível de ansiedade que experimentam frente às atividades (Bandura, 1993; Bzuneck, 2001; Shunk, 1995).

De acordo com Pajares (1996), pesquisas que abordam o senso de auto-eficácia relacionado ao contexto acadêmico sugerem que estudantes com alto senso de auto-eficácia são capazes de realizar tarefas acadêmicas usando mais estratégias cognitivas e metacognitivas e persistem por mais tempo do que aqueles com baixo senso de auto-eficácia. Em contrapartida, crianças que apresentam dificuldades de aprendizagem podem se julgar com baixo senso de auto-eficácia quanto às suas capacidades de desempenharem com sucesso determinadas tarefas acadêmicas.

O constructo auto-eficácia tem sido particularmente aplicado às crianças que experimentam dificuldade de aprendizagem e de comportamento. A auto-eficácia dos estudantes, juntamente com outras crenças e atitudes em relação à aprendizagem, tem sido considerado forte preditor de desempenho acadêmico. A auto-eficácia influencia o desempenho acadêmico e, ao mesmo tempo, é influenciada por este, tendo implicações no desenvolvimento da criança como um todo. Essa influência ocorre tanto por ação direta quanto por seu impacto nos processos de motivação, auto-regulação e autopercepção e nas expectativas de resultados, nas escolhas e interesses (Bandura \& Schunk,1981; Pajares \& Valiante, 1997; Schunk, 1995).

Medeiros, Loureiro, Linhares \& Marturano (2000) estudaram as relações entre o desenvolvimento acadêmico, o senso de auto-eficácia e os aspectos comportamentais de crianças diferenciadas quanto ao desempenho acadêmico. O grupo de crianças com queixa de dificuldade de aprendizagem apresentou um senso de auto-eficácia mais baixo em relação às crianças com bom desempenho, considerando-se pouco competente para a execução com sucesso de atividades acadêmicas específicas.

Segundo Roeser e Eccles (2000), para compreender a relação entre o desempenho acadêmico e saúde mental, resultante da interação de uma série de fatores, é importante diferenciar duas formas de abordagem do desempenho acadêmico: a quantitativa e a qualitativa. Conceituações quantitativas do desempenho acadêmico estão centradas na noção “do que” o estudante faz em situações acadêmicas. O foco na abordagem quantitativa está naqueles comportamentos que podem ser avaliados pelos outros, compreendendo a observação do comportamento das crianças por parte dos pais, professores ou pares. Consideram-se como indicadores quantitativos do desempenho comportamentos tais como o desempenho em sala de aula, o resultado de avaliações padro- nizadas, o investimento de esforço e tempo em tarefas e a escolha de trabalhos desafiadores.

Por outro lado, segundo os autores, os indicadores qualitativos do desempenho consideram “o porquê” os estudantes fazem o que eles fazem. O foco na abordagem qualitativa do desempenho está na avaliação dos processos, tais como as crenças das crianças sobre as causas do sucesso ou fracasso escolar, suas percepções quanto às próprias habilidades para as aprendizagens específicas, suas metas, competências acadêmicas, além das estratégias metacognitivas. Em uma perspectiva qualitativa a atenção está voltada para a compreensão do processo e sua associação ao comportamento manifesto.

Adotando a abordagem conceitual dos indicadores quantitativos e qualitativos do desempenho acadêmico, descrita por Roeser e Eccles (2000), interessou-nos aplicar tais conceitos na avaliação do comportamento de crianças com queixa de dificuldade de aprendizagem e de crianças com bom desempenho.

Considerou-se como indicadores quantitativos do desempenho acadêmico a avaliação da produção e do comportamento das crianças observados clinicamente frente a uma situação orientada para aprendizagem, utilizando-se materiais lúdicos e pedagógicos. Os indicadores qualitativos serão avaliados com base nas percepções das crianças sobre seu próprio desempenho em atividades específicas.

Buscou-se estudar, de forma sistemática, o que as crianças estão produzindo e como estão se comportando e relacionar este padrão de funcionamento comportamental com sua percepção de auto-eficácia.

O objetivo, portanto, deste estudo, consiste na avaliação de aspectos quantitativos e qualitativos do desempenho acadêmico de dois grupos de crianças, relacionando os indicadores quantitativos do comportamento, identificados com base na avaliação da produção e comportamentos orientados para aprendizagem das crianças, e indicadores comportamentais qualitativos, identificados com base nas percepções quanto ao senso de auto-eficácia. Para a avaliação sistemática da produção e do comportamento da criança propõe-se a aplicação de um procedimento, envolvendo a realização de uma observação clínica comportamental frente a uma situação orientada para aprendizagem, utilizando-se materiais lúdicos e acadêmicos e o preenchimento de um Roteiro de Observação Clínica Comportamental da Criança, construído para a finalidade deste estudo.

\section{Método}

\section{Participantes}

Foram avaliadas 52 crianças de ambos os sexos, na faixa etária entre 8 anos e 11 anos e 11 meses, alunos de $1^{\underline{a}}$ a $4^{\underline{a}}$ série de 20 escolas da rede pública do município de Ribeirão Preto (SP), distribuídas em dois grupos:

Grupo 1: Composto por 26 crianças (16 meninos e 10 meninas) encaminhadas ao Ambulatório de Psicologia Clínica Infantil do Hospital das Clínicas da Faculdade de Medicina de Ribeirão Preto - USP, com queixa de dificuldade de aprendizagem. 
Grupo 2: Composto por 26 crianças com bom desempenho acadêmico que freqüentavam um centro escolar: Centro de Atendimento Integral a Crianças e Adolescentes (CAIC).

Para a seleção dos 52 participantes foram inicialmente avaliadas 88 crianças, sendo 36 procedentes do ambulatório e 52 do CAIC. Foram excluídas do grupo 1, dez crianças, e do grupo 2, 26 crianças que apresentaram desempenho abaixo do nível intelectual médio inferior na avaliação intelectual através das Matrizes Progressivas Coloridas de Raven - Escala Especial MPC (normas brasileiras de Angelini, Alves, Custódio \& Duarte, 1987) e aqueles que apresentaram história de tratamento neurológico e/ou psicológico no último ano. Considerou-se como condição para inclusão no G2 o desempenho no Teste de Desempenho Escolar - TDE (normas brasileiras de Stein, 1994), nas áreas de leitura e escrita, compatível com sua faixa etária e/ou série escolar, e a avaliação da professora através do Questionário para Caracterização do Desempenho e do Comportamento da Criança no Ambiente Escolar - DCCE (Machado, Figueiredo, \& Selegato, 1989), indicando bom desempenho.

Na composição do G2 tomaram-se como referência as crianças do G1, buscando-se o balanceamento com relação a sexo, idade da criança e nível de escolaridade dos pais. Após a seleção dos participantes, procedeu-se à comparação dos grupos através do teste não-paramétrico de Mann-Whitney, quanto às variáveis idade (G1/Média (M) = 9 anos e G2/M= 9 anos e 1 mês), escolaridade das mães (G1/M= Sexta série e G2/M= Sétima série), escolaridade dos pais (G1/M= Quarta série e G2/M= Quinta série); não foram observadas diferenças estatisticamente significativas entre os grupos quanto a essas variáveis. Quanto ao nível intelectual das crianças, observou-se diferença estatisticamente significativa entre os grupos sendo que o G1 apresentou um nível intelectual inferior ao G2, medido em termos de percentil ( $\mathrm{G} 1 / \mathrm{M}=44,81$ e $\mathrm{G} 2 / \mathrm{M}=57,88$ ).

O projeto foi apreciado e aprovado pela Comissão de Ética do hospital-escola e pelas Secretarias Municipais do Bem Estar Social e da Educação de Ribeirão Preto. Após a explicação relativa aos objetivos do trabalho e o tipo de participação requerida, foi solicitado aos pais que assinassem o Termo de Consentimento, autorizando a participação das crianças. Após essa etapa as crianças foram convidadas a participar de forma voluntária.

\section{Materiais e instrumentos}

Foram utilizados os seguintes materiais para a realização da observação: lápis sem ponta, caneta, borracha, apontador, régua, livros, folhas sulfite, folhas com pauta, papel quadriculado 10 X $10 \mathrm{~cm}$ coloridos, revistas, miniaturas de animais, carros e blocos de madeira (círculo, quadrado, retângulo e triângulo), gravador e fitas cassetes.

Para a coleta de dados foram utilizados os seguintes instrumentos:

a) Roteiro de Observação Clínica Comportamental da Criança orientada para a aprendizagem, baseado nas proposições de Visca (1987). (Apêndice A).

b) Roteiro de Avaliação do Senso de Auto-eficácia (Medeiros et al., 2000). Esse roteiro focaliza a autopercepção da criança quanto ao seu desempenho acadêmico frente à atividade específica e sua capacidade de realização. Trata-se de procedimento elaborado a partir dos estudos de Bandura (1993) e Shunck (1995). (Apêndice B).

\section{Procedimento}

As crianças foram avaliadas individualmente, as do G1 no Ambulatório e as do G2 no CAIC. Na primeira sessão procedeu-se à aplicação do MPC - Raven Infantil - escala especial, conforme recomendações técnicas.

Em uma segunda sessão, após um breve rapport, procedeu-se à realização da observação utilizando-se para tal um procedimento que inclui atividades lúdicas e pedagógicas e intervenções da examinadora previamente delineadas de forma a assegurar certa sistematização à situação, conforme descrição apresentada a seguir.

Diante de uma diversidade de materiais lúdicos e pedagógicos, pede-se à criança que mostre o que já sabe fazer, o que aprendeu, e o que as pessoas lhe ensinaram. A seguir, indicando o material dentro da caixa: "Este material é para que você use, se precisar, para mostrar-me o que falei que queria saber de você”. Em face dessa proposta, a criança poderá reagir de diferentes formas como falar, ficar paralisada, ignorar o material, entre outras.

Iniciada a realização da observação, com a instrução inicial acima, a examinadora pode adicionar intervenções para facilitar a iniciativa de participação da criança, assim como pode sugerir a transição de uma atividade devidamente demonstrada pela criança para uma nova atividade. As intervenções ou manobras da examinadora podem ser de quatro tipos: (a) resposta gestual (qualquer gesto ou expressão da examinadora que tenha por sentido mobilizar a criança: aceno da cabeça, sorriso, gesticulação, tais como apontar); (b) proposição verbal aberta para demonstrar outra atividade (“Agora eu gostaria que você me mostrasse outra coisa que aprendeu, que sabe ou que lhe ensinaram”); (c) proposição verbal fechada para demonstrar outra atividade ("Você já me mostrou como desenha, agora eu gostaria que você me mostrasse outra coisa qualquer que não seja desenhar"); (d) modelo de alternativa múltipla ("Você pode desenhar, escrever, ler, fazer alguma coisa de matemática ou qualquer outra coisa que lhe venha à cabeça”). As sessões foram gravadas em fitas cassetes e durante a realização da observação clínica a examinadora realizava anotações das observações do comportamento da criança para complementar o registro das sessões.

Após a realização da observação clínica, de duração média de 30 minutos, aplicou-se o Roteiro de Avaliação do Senso de Auto-eficácia. Os itens do Roteiro foram apresentados através de fita cassete gravada, evitando assim variações de entonações e pausas na leitura dos mesmos. As crianças podiam ouvir os itens tantas vezes quantas sentissem necessidade, sempre através do gravador. A entrevistadora, presente na situação, apenas assinalava a opção da criança na folha de respostas.

\section{Tratamento dos dados}

São apresentadas a seguir as etapas cumpridas para a codificação e quantificação dos dados do Roteiro de Obser- 
vação Clínica Comportamental da Criança e do Roteiro de Avaliação do Senso de Auto-eficácia. Após a quantificação, os dados foram submetidos à análise estatística para a comparação entre os grupos de participantes e verificação de associações entre variáveis.

\section{Roteiro de observação clínica comportamental da criança}

Após a conclusão da coleta de dados referente às entrevistas das 52 crianças estudadas, procedeu-se à transcrição literal das fitas cassetes. A transcrição foi complementada com anotações das observações realizadas pela examinadora durante a sessão de avaliação.

Procedeu-se à codificação do material transcrito através da aplicação do Roteiro de Observação Clínica Comportamental da Criança. Definiu-se um procedimento para a codificação com as seguintes etapas: a) ouvir a fita gravada, b) ler o relato transcrito da sessão, c) preencher o roteiro, tendo como suporte as descrições de cada categoria apresentadas no Anexo 1 e o guia de pontuação apresentado a seguir.

A categoria "Produção" compreende três alternativas de resposta, A, B ou C, para cada atividade desenvolvida pela criança avaliada. Para o Nível A foi atribuído um escore 3, para o Nível B foi atribuído um escore 2 e para o Nível C um escore 1. Em seguida, avalia-se o "Desempenho Específico" que compreende quatro alternativas de resposta com gradação progressiva de adequação à situação. As quatro subcategorias, "Reações frente à manobra”, “Temática”, “Comunicação" e "Interação”, dispõem também de alternativas A, B, C, ou D, sendo atribuído escore 3, 2, 1 ou 0 (zero), respectivamente. A categoria "Desempenho Específico” pode ainda ser avaliada quando ocorre a ausência de atividade, ou seja, após uma manobra da examinadora a criança recusa-se a realizar qualquer atividade, porém, não deixa de apresentar as demais subcategorias de reação frente à manobra, temática, comunicação e interação.

A categoria do "Desempenho Geral” compõe-se de duas subcategorias, "Recursos e Manifestações Afetivas”. Quanto aos "Recursos", os itens organização, planejamento e atenção são avaliados de acordo com três alternativas, que além de identificar a presença ou ausência do comportamento, apresentam graduação de freqüência para aquele comportamento: “freqüentemente”, “ocasionalmente”, “não”, “não observado”, sendo atribuído escore 3, 2, 1 e 0 (zero), respectivamente. Para os itens da subcategoria "Manifestações Afetivas” tem-se três alternativas, a saber: A (alternativa que descreve o comportamento com polaridade positiva), B (alternativa que descreve o comportamento com polaridade negativa) ou $\mathrm{C}$ (quando nenhum dos comportamentos é observado), sendo atribuído escore 2, 1 ou 0 (zero), respectivamente.

Para os objetivos deste estudo os roteiros relativos à observação das 52 crianças incluídas no estudo foram preenchidos independentemente por duas examinadoras com experiência prévia em avaliação e intervenção psicopedagógica de crianças com queixa de dificuldade de aprendizagem. As respostas assinaladas no roteiro foram comparadas e procedeu-se ao cálculo dos índices de concordância simples. O índice de concordância geral obtido foi de 76,55 \%. Os desacordos foram discutidos em conjunto pelas duas primeiras autoras e consideram-se as avaliações de consenso pós-discussão para a inclusão na análise geral dos dados.

\section{Roteiro de avaliação do senso de auto-eficácia}

Para a classificação dos dados relativos ao Roteiro de Avaliação do Senso de Auto-eficácia, as respostas foram pontuadas em 0 (zero) ou 1 (um), de acordo com a alternativa escolhida. Os participantes dispunham de duas alternativas de respostas, “sim” ou “não”. Para as respostas favoráveis a um senso de auto-eficácia positivo foi atribuído valor 1 (um), que pode corresponder a um sim ou não dependendo do conteúdo do item em questão. No conjunto de 20 itens, nas dez afirmativas positivas a resposta "sim" é pontuada com valor 1 (um) e nas dez afirmativas negativas a resposta "não” é pontuada com valor 1 (um).

Ex.: "Eu tenho me saído bem" Sim = 1 e Não $=0$

"Eu quero parar de estudar logo" Sim $=0$ e Não $=1$

\section{Tratamento estatístico}

Os dados relativos ao Roteiro de Observação Clínica Comportamental da Criança, especificamente as categorias Produção, Desempenho Específico e Desempenho Geral, e ao Roteiro de Avaliação do Senso de Auto-eficácia foram quantificados e os grupos comparados através do teste nãoparamétrico de Mann-Whitney para amostras independentes $(p \leq 0,05)$.

Para determinar a existência de possíveis associações entre os resultados obtidos através dos referidos procedimentos de avaliação usou-se o coeficiente de correlação nãoparamétrica de Spearman $(p \leq 0,05)$.

\section{Resultados}

Os resultados estão organizados em três tópicos. No primeiro tópico, serão apresentados os indicadores quantitativos obtidos através da análise dos dados referente ao Roteiro de Observação Clínica Comportamental da Criança. No segundo, serão apresentados os indicadores qualitativos obtidos através da análise do Roteiro de Avaliação do Senso de Auto-eficácia. No terceiro tópico, serão apresentadas as associações entre os resultados alcançados nas duas avaliações realizadas.

\section{Indicadores quantitativos - Roteiro de Observação Clínica Comportamental da Criança.}

Os dados relativos ao Roteiro de Observação Clínica Comportamental da Criança orientada para aprendizagem foram analisados discriminando-se as três categorias: Produção, Desempenho Específico e Desempenho Geral.

Com base nas informações do Roteiro de Observação Clínica Comportamental da Criança, pôde-se realizar um levantamento do número de atividades realizadas pelas crianças nos dois grupos. As crianças do G1 apresentaram o total de 160 atividades e as crianças do G2, o total de 127. A análise geral da categoria Produção permitiu observar que das 160 atividades desenvolvidas pelas crianças do G1, 42\% foram 
atividades de qualidade média, 39\% de qualidade baixa e 19\% alta, enquanto que das 127 atividades realizadas pelas crianças do G2, 44\% foram atividades de qualidade alta, $28 \%$ qualidade média e $28 \%$ de qualidade baixa.

A fim de comparar os grupos, foram realizadas análises das categorias Produção, Desempenho Específico e Desempenho Geral considerando-se o número total de atividades de cada grupo avaliado. A Tabela 1 apresenta a comparação dos grupos, discriminando os itens que apresentaram diferença estatisticamente significativa.

Nota-se na Tabela 1 que os grupos apresentaram diferença estatisticamente significativa em seis itens do Roteiro de Observação Clínica Comportamental da Criança. Quanto à categoria "Produção", observou-se que os grupos apresentaram diferenças estatisticamente significativas para as atividades de qualidade média, em que o G1 apresentou média de desempenho mais alta para tais atividades quando comparado ao G2. Apesar de não se observar diferenças estatisticamente significativas nos outros dois níveis, é interessante notar que o G1 apresenta média mais alta que o G2 para as atividades de qualidade baixa, e uma média mais baixa que o G2 para as atividades de qualidade alta. As atividades desenvolvidas pelo G1 são categorizadas com nível de qualidade médio, com tendência a baixo.

Na categoria “Desempenho Específico”, as subcategorias Reações frente à manobra, Comunicação e Interação apresentaram itens que diferenciaram os grupos. Observou-se que, quanto às Reações frente à manobra da examinadora, o G1 apresentou média mais alta do que o G2 para o item espontaneamente realiza atividade; sem que a examinadora verbalizasse. Na subcategoria Comunicação, o G1 diferenciou-se em relação ao G2 quanto a não realizar atividades e se comunicar, ou seja, as crianças do G1 distanciaram-se mais da realização de atividades enquanto verbalizaram idéias e fatos quando comparadas às crianças do G2. Quanto à Interação, o
G1 diferenciou-se quanto a interagir adequadamente com a examinadora sem estimulação, apresentando média mais alta que o G2.

Observa-se que o G1, tanto para a realização das atividades quanto para a interação, apresentam um padrão comportamental independente da verbalização da E. Realizam as atividades e buscam a interação com a E. de forma espontânea; entretanto distanciam-se da realização da atividade para a interação e comunicação. Observa-se que o padrão comportamental de espontaneidade para a comunicação e interação implica em um distanciamento da realização da atividade. Ou seja, as crianças com queixa de dificuldade de aprendizagem preferem interagir e conversar a realizarem as atividades.

Quanto à Temática, os grupos não se diferenciaram estatisticamente. Os grupos apresentaram diversidade de temas que variava desde temas sobre atividades acadêmicas a temas tais como família, amigos ou brincadeiras.

No que se refere ao "Desempenho Geral”, dividido em duas subcategorias, Recursos e Manifestações Afetivas, notou-se que, quanto aos Recursos, o G1 apresentou média significativamente mais baixa quanto aos itens organização e atenção quando comparado ao G2. Considerando-se o escore total e ao item planejamento da subcategoria Recursos não se observou diferença estatisticamente significativa entre os grupos. Quanto às Manifestações Afetivas os grupos não se diferenciaram significativamente com relação a julgamento, decisão, iniciativa, ansiedade, auto-regulação e ao escore total.

Indicadores qualitativos - Roteiro de Avaliação do Senso de Auto-eficácia

Serão apresentados os dados da avaliação do senso de auto-eficácia com relação ao escore total. Os resultados relativos ao Roteiro de Avaliação do Senso de Auto-eficácia quan-

Tabela 1

Observação do Comportamento da Criança Orientado para a Aprendizagem - itens com diferença estatisticamente significativa na comparação dos grupos de crianças com queixa de dificuldade de aprendizagem (G1) e crianças com bom desempenho acadêmico (G2)

\begin{tabular}{|c|c|c|c|c|c|c|c|}
\hline Categoria & & Itens & $\mathrm{G}$ & Média & $d p$ & Mediana & $p$ \\
\hline Produção & & Qualidade média & 1 & 2,62 & 2,30 & 2,00 & 0,05 \\
\hline & & & 2 & 1,50 & 1,68 & 1,00 & \\
\hline Desempenho & Reações frente à & Espontaneamente realiza & 1 & 1,92 & 2,17 & 1,50 & 0,003 \\
\hline Específico & manobra & atividade & 2 & 0,42 & 0,86 & 0,00 & \\
\hline & Comunicação & Não realiza atividades e se & 1 & 1,92 & 2,08 & 1,00 & 0,002 \\
\hline & & comunica & 2 & 0,69 & 1,54 & 0,00 & \\
\hline & Interação & Sem estimulação - interação & 1 & 2,39 & 2,48 & 2,50 & 0,03 \\
\hline & & adequada & 2 & 0,89 & 1,53 & 0,00 & \\
\hline Desempenho & Recursos & Organização & 1 & 2,15 & 1,08 & 3,00 & 0,05 \\
\hline Geral & & & 2 & 2,69 & 0,68 & 3,00 & \\
\hline & & Atenção & 1 & 2,69 & 0,55 & 3,00 & 0,02 \\
\hline & & & 2 & 2,96 & 0,20 & 3,00 & \\
\hline
\end{tabular}

(Teste não-paramétrico de Mann-Whitney) 
Tabela 2

Percepção de Auto-eficácia frente ao desempenho acadêmico - média, desvio padrão e mediana do escore total na comparação dos grupos de crianças com queixa de dificuldade de aprendizagem (G1) e crianças com bom desempenho acadêmico (G2)

\begin{tabular}{ccccc}
\hline Grupos & Média & Dp & Mediana & $p$ \\
\hline G1 & 12,19 & 4,22 & 12,50 & 0,001 \\
G2 & 18,54 & 2,16 & 19,00 & \\
\hline
\end{tabular}

(Teste não-paramétrico de Mann-Whitney)

to à comparação do escore total de cada grupo estão apresentados na Tabela 2.

Observou-se diferença significativa entre os dois grupos na avaliação do escore total, considerando-se $\mathrm{p}<0,001$. O G1 obteve pontuação menor que o G2, caracterizando uma avaliação mais baixa do senso de auto-eficácia por parte das crianças com queixa de dificuldade de aprendizagem, denotando autopercepções negativas com relação a si.

\section{Correlações entre Nível Intelectual, o Senso de Auto-eficácia e o Desempenho Geral na Observação Clínica Comportamental da Criança.}

Quanto à associação entre as variáveis senso de autoeficácia e nível intelectual das crianças, investigado através do coeficiente de correlação não-paramétrica de Spearman, não se observou correlação significativa entre elas.
As correlações entre as variáveis Senso de Auto-eficácia e as subcategorias do Desempenho Geral de Recursos e Manifestações Afetivas, relativas aos dois grupos, estão apresentadas na Tabela 3.

Observou-se que, no G1, o senso de auto-eficácia correlacionou-se com o escore total da subcategoria Manifestações afetivas, assim como com os itens iniciativa e decisão. Esses dados sugerem que quanto mais alto o senso de auto-eficácia da criança, mais positivas são as manifestações afetivas especificamente quanto a maior capacidade de iniciativa (ativa e independente) e de decisão (decidida e capacidade de escolha). No G2, o senso de auto-eficácia correlacionou-se significativamente apenas com o item autojulgamento, indicando que quanto mais alto o senso de auto-eficácia, mais positivo o julgamento com relação ao seu

Tabela 3

Associações entre a Percepção do Senso de Auto-eficácia, e a avaliação geral dos Recursos e as Manifestações Afetivas identificadas nos grupos de crianças com queixa de dificuldade de aprendizagem (G1) e crianças com bom desempenho acadêmico (G2)

\begin{tabular}{|c|c|c|c|c|c|c|c|c|c|}
\hline & $\mathrm{G}$ & $\mathrm{AE}$ & $\mathrm{R}$ & ma-t & ma-j & ma-i & ma-d & ma-a & ma-r \\
\hline \multirow[t]{2}{*}{ Auto-eficácia } & 1 & 1,00 & 0,14 & $0,50^{*}$ & 0,29 & $0,62 *$ & $0,57 *$ & 0,29 & $-0,06$ \\
\hline & 2 & 1,00 & 0,21 & 0,29 & $0,49 *$ & $-0,01$ & 0,18 & 0,17 & 0,23 \\
\hline \multirow[t]{2}{*}{ Recursos } & 1 & & 1,00 & $0,41 *$ & $-0,02$ & 0,25 & 0,27 & 0,07 & $0,61 *$ \\
\hline & 2 & & 1,00 & $0,44^{*}$ & 0,31 & 0,19 & $0,35^{*}$ & 0,26 & $0,62 *$ \\
\hline \multirow[t]{2}{*}{ Manifestação afetiva - Total } & 1 & & & 1,00 & $0,54 *$ & $0,75^{*}$ & $0,84 *$ & $0,66^{*}$ & $0,56^{*}$ \\
\hline & 2 & & & 1,00 & $0,60 *$ & $0,79 *$ & $0,88^{*}$ & $0,79 *$ & $0,64 *$ \\
\hline \multirow[t]{2}{*}{ Julgamento } & 1 & & & & 1,00 & 0,27 & $0,35^{*}$ & 0,22 & 0,09 \\
\hline & 2 & & & & 1,00 & 0,25 & $0,51 *$ & 0,26 & 0,20 \\
\hline \multirow[t]{2}{*}{ Iniciativa } & 1 & & & & & 1,00 & $0,75^{*}$ & $0,39 *$ & 0,09 \\
\hline & 2 & & & & & 1,00 & $0,77^{*}$ & $0,57 *$ & $0,41 *$ \\
\hline \multirow[t]{2}{*}{ Decisão } & 1 & & & & & & 1,00 & $0,46^{*}$ & 0,29 \\
\hline & 2 & & & & & & 1,00 & $0,65^{*}$ & $0,41 *$ \\
\hline \multirow[t]{2}{*}{ Ansiedade } & 1 & & & & & & & 1,00 & 0,26 \\
\hline & 2 & & & & & & & 1,00 & $0,43 *$ \\
\hline \multirow[t]{2}{*}{ Auto-regulação } & 1 & & & & & & & & 1,00 \\
\hline & 2 & & & & & & & & 1,00 \\
\hline
\end{tabular}

(Matriz de correlação não-paramétrica de Spearman)

$\mathrm{G}=$ grupos; $\mathrm{AE}=$ auto-eficácia; $\mathrm{R}=$ Recursos; $\mathrm{ma}-\mathrm{t}=$ Manifestações Afetivas escore total; $\mathrm{ma}-\mathrm{j}=$ julgamento; ma-i = iniciativa; ma- $\mathrm{d}=$ decisão; ma-a= ansiedade; ma-r = regulação.

$* \mathrm{p} \leq 0,05$ 
próprio desempenho. As crianças com queixa de dificuldades de aprendizagem quando apresentam um senso de autoeficácia mais positivo manifestam comportamentos concomitantes de iniciativa e decisão, entretanto não verbalizam julgamentos positivos em relação à si mesmas, como as crianças com bom desempenho fazem. O G2, quando apresenta auto-eficácia positiva verbaliza, durante a realização das atividades, julgamentos positivos sobre si mesmo.

A subcategoria Recursos escore total apresentou-se associada, no G1, ao escore total das Manifestações Afetivas e à capacidade de auto-regulação. Indica que quanto maior a presença de planejamento, organização e atenção, maior a capacidade de auto-regulação e mais positivas as manifestações afetivas. No G2, observou-se que a subcategoria Recursos apresentou-se associada ao escore total das Manifestações Afetivas, à capacidade de auto-regulação e decisão. Indica que quanto mais recursos na criança, mais positivas são as manifestações afetivas e maior a capacidade auto-regulação e decisão. Observa-se que, para os dois grupos, a capacidade de auto-regulação aparece associada às manifestações de planejamento, atenção e organização. Podemos pensar que o controle dos impulsos pode favorecer à manifestação das capacidades de planejamento, organização e atenção durante à realização da tarefa.

Quanto ao escore total da subcategoria Manifestações Afetivas, em ambos os grupos, notam-se correlações em relação a todos os itens que compõem essa subcategoria, ou seja, quanto mais positivas as manifestações afetivas, mais positivos o autojulgamento, maior a capacidade de iniciativa, decisão e auto-regulação e mais baixo o nível de ansiedade.

Quando se avaliam as correlações entre os itens da subcategoria Manifestações Afetivas, observou-se que tanto para o G1 quanto para o G2, as correlações foram semelhantes para os seguintes indicadores: autojulgamento correlacionou-se com indicadores de decisão; manifestações de iniciativa correlacionou-se com indicadores de decisão e nível de ansiedade; manifestações de decisão correlacionouse com nível de ansiedade. Entretanto, observou-se que no G2, além das correlações apontadas acima, o indicador de auto-regulação correlacionou-se com os indicadores de iniciativa, decisão e nível de ansiedade. Novamente observa-se que a capacidade de auto-regulação aparece associada à manifestações comportamentais positivas como iniciativa, decisão e baixo nível de ansiedade. O fato de esta associação ser significativa somente para o grupo com bom desempenho, faz-nos pensar o quanto estas capacidades podem estar favorecendo um melhor aproveitamento escolar.

\section{Discussão}

Com base nos indicadores quantitativos do desempenho acadêmico das crianças com relação à produção e comportamento orientado para aprendizagem, pode-se traçar um perfil do padrão comportamental de crianças com queixa de dificuldades de aprendizagem em comparação às crianças com bom desempenho escolar. Observa-se que as primeiras, quando comparadas às segundas, contrariando a hipótese inicial com relação à sua capacidade de realização, apresentaram um maior número de atividades, com padrão predominantemente médio de qualidade e apresentaram-se mais espontâneas para a realização das atividades e para a interação. Em contrapartida, denotaram poucos recursos de organização e atenção para aprimorarem a qualidade de suas produções e adequarem seu padrão de interação.

Analisando-se o indicador qualitativo expresso através das percepções das crianças, observa-se que o grupo com queixa de dificuldade de aprendizagem apresentou indicadores de senso de auto-eficácia mais rebaixado em relação às crianças com bom desempenho escolar, considerando-se pouco competentes para a execução com sucesso de atividades acadêmicas específicas. Esse padrão de funcionamento é concordante com dados de outros estudos que apontam as relações entre baixo senso de auto-eficácia e baixo desempenho acadêmico (Pajares, 1996; Pajares \& Valiante, 1997, Schunk, 1995).

Integrando-se os indicadores quantitativos e qualitativos do desempenho acadêmico verifica-se que apesar de as crianças com dificuldade de aprendizagem realizarem produções de qualidade média e buscarem de forma mais espontânea a interação e comunicação, julgavam-se com baixa capacidade para desempenhar com sucesso as atividades acadêmicas, o que possivelmente reflete suas autopercepções que têm por base a sua história prévia de baixo rendimento acadêmico, o que justificou inclusive seu encaminhamento para atendimento psicológico.

Crianças que apresentam baixo senso de auto-eficácia em um dado domínio tendem a evitar tarefas difíceis, percebendo-as como ameaça pessoal; apresentam baixas aspirações e envolvimento com metas mantendo o foco mais no autodiagnóstico do que em como ter boa realização e desempenho. Diante de tarefas difíceis, geralmente, fixam-se muito mais em suas deficiências pessoais, nos obstáculos que irão encontrar e todos os tipos de resultados aversivos. Pessoas que se percebem como ineficazes tendem a afrouxar seus esforços e fugirem rapidamente de situações de dificuldade. Em contrapartida, alto senso de auto-eficácia realça a realização pessoal em muitos campos.

Bandura (1986) considera que os julgamentos de autoeficácia atuam como mediadores entre os fatores reconhecidos que influenciam o comportamento, tais como aptidões, conhecimentos, realizações prévias e habilidade e o comportamento subseqüente. A realização pessoal requer tanto a capacidade, quanto a crença na auto-eficácia para usá-la da melhor forma (Bzuneck, 2001).

Schunk (1995) refere que crianças com baixo rendimento apresentam baixo senso de auto-eficácia, são mais passivas, usam menos estratégias cognitivas e metacognitivas e apresentam falhas no processo de auto-regulação, quando comparadas com seus pares com bom rendimento. Os dados encontrados no presente estudo corroboram o padrão comportamental das crianças com baixo rendimento, descritos pelo autor acima. Exceção a isto foi o achado referente à passividade das crianças, na medida em que as crianças com 
queixa de dificuldade de aprendizagem apresentaram um padrão ativo e com iniciativa, tanto para a interação quanto para a realização de atividades; diferente das crianças com bom desempenho, que estabeleceram um padrão mais reservado e inibido.

Analisando-se os resultados da categoria Produção, observa-se que as crianças com queixa de dificuldade de aprendizagem realizaram maior número de atividades, porém, em nível baixo de qualidade, quando comparadas às crianças com bom desempenho. Pode-se pensar que essas crianças estavam muito mais preocupadas em "produzir em quantidade" do que "produzir com qualidade". A orientação da atenção dessas crianças parecia mais voltada para si do que para a realização da tarefa. O padrão mais ativo e com iniciativa apresentado pelas crianças com queixas de dificuldades de aprendizagem, com relação à interação e produção, podem ser indicativos de condutas relacionadas à baixa capacidade de auto-regulação apresentada pelas crianças deste grupo.

Marturano, Linhares e Parreira (1993) encontraram em seu estudo que as crianças com dificuldades de aprendizagem apresentam modos de enfrentamento inadequados frente às situações cotidianas e às relações interpessoais, predominando condutas indicativas de baixa capacidade de autoregulação, hostilidade e resistência a normas, assim como indícios de estados subjetivos negativos, com tendência à generalização de tais reações.

O perfil de espontaneidade e disponibilidade para a comunicação poderia ser considerado como um aspecto positivo com relação a essas crianças, se não estivesse associado à baixa capacidade de recursos quanto a organização e atenção, auto-regulação e baixo senso de auto-eficácia. Pode-se pensar no quanto essa associação de indicadores se relaciona com a dificuldade de obtenção de sucesso em tarefas acadêmicas, reforçando o chamado "círculo vicioso do fracasso” (Marturano et al., 1993). Neste a criança percebe-se como pouco eficaz para realizar atividades com sucesso, conta com poucos recursos e baixo nível de estratégias auto-regulatórias, favorecendo desempenho pouco desejável, que por sua vez confirma sua crença de baixa eficácia e assim por diante.

Por outro lado, considerando-se o contexto de avaliação diagnóstica em que os dados foram colhidos, precedendo o atendimento psicológico, pode-se interpretar que a disposição para interagir e para produzir refletem também um comportamento sociável destas crianças favorecedor ao suporte externo.

Segundo Pajares e Valiante (1997), o processo de criação e aplicação das crenças de uma pessoa se dão quando os indivíduos engajam-se em comportamentos, interpretam os resultados de suas ações, usam estas interpretações para desenvolver crenças sobre suas capacidades para engajaremse em comportamentos subseqüentes de um mesmo domínio e se comportam de acordo com a crença criada. Na escola, por exemplo, as crenças que os estudantes desenvolvem sob suas capacidades acadêmicas ajudam a determinar o que eles fazem com o conhecimento e as habilidades que estão adquirindo.
Destaca-se que, através da aplicação do Roteiro de Observação Clínica Comportamental da Criança, foram elucidados aspectos relevantes do desempenho acadêmico, permitindo a identificação de recursos e limites das crianças frente a uma situação orientada para aprendizagem. O Roteiro poderá ser utilizado como uma forma de sistematização da observação individualizada de crianças com dificuldades de aprendizagem, visto que aborda aspectos diversos do seu funcionamento tais como: nível de qualidade da produção, reação frente à situação, nível de comunicação e interação e recursos disponíveis para a execução de tarefas acadêmicas. Além desses aspectos permite observar as manifestações afetivas das crianças, que dizem respeito a sua capacidade de autojulgamento, iniciativa, decisão, auto-regulação e nível de ansiedade.

A utilização deste Roteiro de avaliação como um procedimento diagnóstico pode favorecer o planejamento de intervenções psicopedagógicas, no âmbito educacional ou terapêutico, que levem em conta tanto as capacidades e recursos potenciais quanto as autopercepções. Neste sentido, com base na análise dos indicadores quantitativos e qualitativos do desempenho acadêmico, pode-se sugerir que o apoio psicopedagógico para as crianças com dificuldades de aprendizagem deve priorizar o favorecimento do desenvolvimento das percepções de auto-eficácia e da auto-regulação.

Segundo Bandura (1993) e Schunk (1995), a crença firme dos estudantes em sua auto-eficácia para manejar as suas próprias atividades de aprendizagem e motivação fornece confiança e realce às realizações de desempenho. Consideram assim que os estudantes devem desenvolver habilidades de regulação da motivação, afetos e determinantes sociais do seu desempenho acadêmico, tão bem quanto os aspectos cognitivos.

Observou-se que, nos dois grupos estudados, quanto maior a presença de recursos, especificamente capacidade de planejamento, organização e atenção, maior a presença de comportamento de controle de impulsos e mais positivas as manifestações afetivas. O grupo de crianças com bom desempenho ainda apresentou correlação entre a presença de recursos com a manifestação de decisão. Nesse sentido, o apoio psicopedagógico pode funcionar para essas crianças como condição favorecedora do desenvolvimento do controle dos impulsos na medida em que oferece condições externas de controle e aprendizagem de padrões comportamentais.

Os estudantes necessitam de um guia social durante a fase inicial de aprendizagem de habilidades complexas para prepará-los para se engajarem em uma prática auto-regulativa eficaz. Quando estratégias de aprendizagem validadas socialmente são modeladas e adotadas como metas que valorizam o processo de aprendizagem, essas levam à prática e automonitoramento. Isso faz com que os estudantes atribuam sucesso às estratégias pessoais controláveis experienciando ganhos em percepções de sua auto-eficácia e de motivação intrínseca o que favorece buscar outras habilidades futuras. 
Por outro lado, as intervenções psicopedagógicas com crianças com dificuldades de aprendizagem constituem-se em um desafio pela multiplicidade de fatores individuais e ambientais que atuam no curso de desenvolvimento de uma criança. O procedimento utilizado, o Roteiro de Observação Clínica Orientado para aprendizagem, não permite estabelecer relações pontuais relativas ao senso de auto-eficácia, o comportamento e o rendimento acadêmico, mas permitiu a identificação de recursos e dificuldades que podem favorecer no planejamento de um processo de intervenção psicopedagógico.

A avaliação do senso de auto-eficácia no contexto acadêmico pode ser de utilidade para o planejamento de procedimentos de intervenção. Um aumento evidente no modo como os estudantes se percebem no nível acadêmico afeta seu desempenho e comportamentos acadêmicos subseqüentes (Pajares, 1996).

Como implicação dessa, destaca-se a importância de se oferecer às crianças ferramentas que lhes permitam, além da aquisição de habilidades, desenvolver crenças mais positivas em relação às suas próprias capacidades de realização. Sugere-se que no trabalho com crianças na fase inicial de aprendizagem formal seja valorizado o desenvolvimento da auto-eficácia como recurso favorecedor do processo de aprendizagem.

Outra implicação desta análise sugere a importância de se manter crenças motivacionais mesmo quando a realização é baixa. Entre baixos realizadores, aqueles que tinham um senso de auto-eficácia alta, expectativa de resultado mais positiva, visão moderada da influência causal da inteligência e baixa atribuição para sorte, como causa do sucesso, apresentaram realizações mais altas. Tal afirmativa chama a atenção para a presença de padrões intra-individuais múltiplos, ou seja, combinações de motivação e cognição em doses diferentes podem levar aos mesmos resultados de realização. Compreender esses diferentes padrões favorece projetos de intervenção mais adequados aos perfis encontrados.

Neste sentido, compreendendo o dinamismo das múltiplas variáveis envolvidas no comportamento de crianças orientado para a aprendizagem, por meio da observação sistemática de crianças e da autopercepção pode-se destacar a importância do senso de auto-eficácia como variável mediadora do desempenho acadêmico, influenciando a produção e a interação.

\section{Agradecimento}

As autoras agradecem à FAPESP e ao CNPq pelo apoio.

\section{Referências}

Angelini, A., Alves, I., Custódio, E., \& Duarte, W. (1987). Manual das matrizes progressivas coloridas Raven. São Paulo: Casa do Psicólogo.

Bandura, A. (1982). Self-efficacy mechanism in human agency. American Psychologist, 37, 122-147.

Bandura, A. (1993). Perceived self-efficacy in cognitive development and functioning. Educational Psychologist, 28, 117-148.

Bandura, A., \& Schunk, D. H. (1981). Cultivating competence, self-efficacy, and intrinsic interest through proximal self-motivation. Journal of Personality and Social Psychology, 41, 586-598.

Bronfenbrenner, U. (1996). A ecologia do desenvolvimento humano: experimentos naturais e planejados. Porto Alegre: Artes Médicas.

Bzuneck, J. A. (2001). As crenças de auto-eficácia e o seu papel na motivação do aluno. In J. A. Bzuneck \& E. Boruchovitch (Orgs.), A motivação do aluno: contribuições da Psicologia contemporânea (pp. 116-133). Petrópolis: Vozes.

Machado, V. L. S., Figueiredo, M. A., \& Selegato, M. V. (1989). Caracterização do comportamento de alunos em sala de aula através de escalas de desempenho. Estudos de Psicologia (PUCCAMP), 6, 50-76.

Marturano, E. M., Linhares, M. B. M., \& Parreira, V. L. C. (1993). Problemas emocionais e comportamentais associados a dificuldades na aprendizagem escolar. Medicina, 26, 161-175.

Medeiros, P. C. (2000). Crianças com dificuldades de aprendizagem: avaliação do senso de auto-eficácia e dos aspectos comportamentais. Dissertação de mestrado não-publicada, Universidade de São Paulo, Ribeirão Preto, SP.

Medeiros, P. C., Loureiro, S.R., Linhares, M. B. M., \& Marturano, E. M. (2000). A auto-eficácia e os aspectos comportamentais de crianças com dificuldade de aprendizagem. Psicologia: Reflexão e Crítica, 13, 327-336.

Pajares, F. (1996). Self-efficacy beliefs in academic settings. Review of Educational Research, 66, 543-578.

Pajares, F., \& Valiante, G. (1997). The predictive and mediational role of the writing self-efficacy beliefs of upper elementary students. Journal of Educational Research, 90, 353-360.

Roeser, R.W., \& Eccles, J. S. (2000). Schooling and mental health. In A. J. Sameroff, M. Lewis \& S. M. Miller (Orgs.), Handbook of developmental psychopathology. (pp. 135-156). Nova York: Kluwer/Plenum.

Rutter, M., \& Sroufe, L. A. (2000). Developmental psychopathology: Concepts and challenges. Development and Psychopathology, 12, 265-296.

Sameroff, A. J. (2000). Developmental systems and psychopathology. Development and Psychopathology, 12, 297-312.

Schunk, D. H. (1995). Self-efficacy and education and instruction. In J. E. Maddux (Org.), Self-efficacy, adaptation, and adjustment: Theory, research, and application (pp. 281-301). Nova York: Plenum.

Stein, L. M. (1994). TDE - Teste de desempenho escolar: Manual para aplicação e interpretação. São Paulo: Casa do Psicólogo.

Visca, J. (1987). Clínica psicopedagógica: epistemologia convergente. Porto Alegre: Artes Médicas. 
Paula Cristina Medeiros, psicóloga, especialista em Psicopedagogia, mestre em Saúde Mental, é doutoranda em Psicologia da Faculdade Filosofia Ciências e Letras de Faculdade de Ribeirão Preto, Universidade de São Paulo.

Sonia Regina Loureiro, psicóloga, Professora Doutora da Faculdade de Medicina de Ribeirão Preto-USP e docente nos Cursos de Pós-graduação em Ciências Médicas (área Saúde Mental) e Psicologia, na Universidade de São Paulo/Ribeirão Preto. Coordena o Serviço de Psicodiagnóstico do Hospital de Clínicas de Ribeirão Preto, onde mantém atividades de formação de recursos humanos e de pesquisa com instrumentos e procedimentos de avaliação em diferentes contextos psicossociais.

Maria Beatriz Martins Linhares, psicóloga, especialista em Psicologia Clínica Infantil, mestre em Educação Especial, doutora em Psicologia Experimental, é docente da Faculdade de Medicina de Ribeirão Preto, Universidade de São Paulo, orientadora no Cursos de Pós-graduação em Saúde Mental (FMRP/USP) e Psicologia (FFCLRP/USP). Coordenadora das seções Psicologia do Desenvolvimento na área Saúde e Psicologia Pediátrica do Serviço de Psicologia do Hospital das Clínicas da FMRP.

Edna Maria Marturano, psicóloga, é Professora Titular da Faculdade de Medicina de Ribeirão Preto/USP e docente nos Cursos de Pós-graduação em Ciências Médicas (área Saúde Mental) e Psicologia, na Universidade de São Paulo/Ribeirão Preto. Coordena o Ambulatório de Psicologia Infantil do Hospital de Clínicas de Ribeirão Preto, onde mantém atividades de pesquisa, formação de recursos humanos e desenvolvimento de intervenções preventivas, focalizando crianças que vivem em situação de risco psicossocial e suas famílias. Endereço para correspondência: Rua Iguape, 603, apto. 62, Bairro Jardim Paulista, 14.090-090, Ribeirão Preto, SP. Tel.: (16) 618.1667. E-mail: pmedeiros@netsite.com.br ou srlourei@fmrp.usp.br 


\section{Apêndice A}

Roteiro de observação clínica comportamental da criança

Os itens das categorias Produção e Desempenho Específico devem ser observados e avaliados para cada uma das atividades realizadas pela criança durante a sess ão de avaliação.

\begin{tabular}{|l|c|}
\hline $\begin{array}{l}\text { I Produção: compreende o conjunto de atividades desenvolvidas verbal e motoras, considerando } \\
\text { o nível de adequação e elaboração. }\end{array}$ & Escore \\
\hline A Qualidade alta & 3 \\
\hline B Qualidade média & 2 \\
\hline C Qualidade baixa & 1 \\
\hline $\begin{array}{l}\text { II Desempenho específico: compreende o conteúdo verbal predominante e as formas de } \\
\text { comunicação e interação da criança frente à situação. }\end{array}$ & \\
\hline Reação frente à manobra da examinadora & 3 \\
\hline A Dispensa manobras, e espontaneamente realiza atividade. & 2 \\
\hline B Após manobras, sem estimulação realiza atividade. & 1 \\
\hline C Após manobras, com estimulação realiza atividade. & 0 \\
\hline D Após manobras, com estimulação não realiza atividade & \\
\hline Temática & 3 \\
\hline A Tarefas acadêmicas. & 2 \\
\hline B Escola. & 1 \\
\hline C Família, colegas, amigos e/ou brincadeiras. & 0 \\
\hline D Outros temas (diferentes dos itens b, c, e d) & 3 \\
\hline Comunicação & 2 \\
\hline A Centrada na realização da atividade - fala pertinente ao que está fazendo & 1 \\
\hline B Centrada na realização da atividade - silêncio ou monossílabos & 0 \\
\hline C Distanciando-se da realização da atividade - fala pertinente ao que está fazendo & \\
\hline D Distanciando-se da realização da atividade - silêncio ou monossílabos & 3 \\
\hline Interação & 2 \\
\hline A Sem estimulação responde adequadamente e interage com a examinadora & 1 \\
\hline B Após estimulação verbal responde adequadamente e não prossegue interação. & 0 \\
\hline C Após estimulação verbal responde monossilabicamente à examinadora & \\
\hline D Após estimulação verbal ignora a examinadora & \\
\hline
\end{tabular}

Para cada atividade realizada pela criança, responda os itens das categorias Produção e Desempenho Especifico com a resposta que lhe parece melhor descrever o que você pode observar no comportamento da criança.

\begin{tabular}{|c|c|c|c|c|c|c|c|c|c|c|c|}
\hline \multicolumn{12}{|c|}{ Número de atividades realizadas pela criança: } \\
\hline & & \multicolumn{10}{|c|}{ Produção e Desempenho Específico } \\
\hline & & 1 & 2 & 3 & 4 & 5 & 6 & 7 & 8 & 9 & 10 \\
\hline I & 1. Nível de qualidade & & & & & & & & & & \\
\hline \multirow[t]{4}{*}{ II } & 1. Reação à manobra & & & & & & & & & & \\
\hline & 2. Temática & & & & & & & & & & \\
\hline & 3. Comunicação & & & & & & & & & & \\
\hline & 4. Interação & & & & & & & & & & \\
\hline
\end{tabular}


Os itens da categoria Desempenho Geral devem ser observados e avaliados considerando o conjunto de atividades realizadas durante toda a sessão.

\begin{tabular}{|c|c|c|c|}
\hline \multicolumn{3}{|c|}{ III Desempenho Geral : } & Escore \\
\hline \multicolumn{4}{|c|}{ III. 1 Recursos } \\
\hline \multirow[t]{4}{*}{ Organização } & \multirow[t]{4}{*}{ Organização e cuidado com os materiais } & Freqüentemente & 3 \\
\hline & & Ocasionalmente & 2 \\
\hline & & Não & 1 \\
\hline & & Não observado & 0 \\
\hline \multirow[t]{4}{*}{ Planejamento } & \multirow{4}{*}{$\begin{array}{l}\text { Atem-se as etapas naturais para a realização da } \\
\text { atividade }\end{array}$} & Freqüentemente & 3 \\
\hline & & Ocasionalmente & 2 \\
\hline & & Não & 1 \\
\hline & & Não observado & 0 \\
\hline \multirow[t]{4}{*}{ Atenção } & \multirow[t]{4}{*}{ Voltada para a atividade } & Freqüentemente & 3 \\
\hline & & Ocasionalmente & 2 \\
\hline & & Não & 1 \\
\hline & & Não observado & 0 \\
\hline \multicolumn{4}{|c|}{ III. 2 Manifestações afetivas } \\
\hline \multirow[t]{3}{*}{ Julgamento } & \multicolumn{2}{|l|}{ Positivos sobre si } & 2 \\
\hline & \multicolumn{2}{|l|}{ Negativos sobre si. } & 1 \\
\hline & \multicolumn{2}{|l|}{ Nenhuma das alternativas. } & 0 \\
\hline \multirow[t]{3}{*}{ Iniciativa } & \multicolumn{2}{|l|}{ Ativa, independente } & 2 \\
\hline & \multicolumn{2}{|l|}{ Apática, submissa } & 1 \\
\hline & \multicolumn{2}{|l|}{ Nenhuma das alternativas } & 0 \\
\hline \multirow[t]{3}{*}{ Decisão } & \multicolumn{2}{|l|}{ Decidida, capacidade de escolha } & 2 \\
\hline & \multicolumn{2}{|l|}{ Indecisa, dificuldade de escolha } & 1 \\
\hline & \multicolumn{2}{|l|}{ Nenhuma das alternativas } & 0 \\
\hline \multirow[t]{3}{*}{ Ansiedade } & \multicolumn{2}{|l|}{ Ansiedade inicial que desaparece ao longo da sessão. } & 2 \\
\hline & \multicolumn{2}{|l|}{ Ansiedade inicial e ao longo da sessão } & 1 \\
\hline & \multicolumn{2}{|l|}{ Nenhuma das alternativas } & 0 \\
\hline \multirow[t]{3}{*}{ Auto-regulação } & \multicolumn{2}{|c|}{$\begin{array}{l}\text { Presença de auto-regulação, completa os assuntos/atividades, responde } \\
\text { com adequação ao proposto }\end{array}$} & 2 \\
\hline & \multicolumn{2}{|c|}{$\begin{array}{l}\text { Interrompe os assuntos ou atividades iniciadas, parece não ouvir, responde } \\
\text { de forma inadequada. }\end{array}$} & 1 \\
\hline & \multicolumn{2}{|c|}{ Nenhuma das alternativas } & 0 \\
\hline
\end{tabular}

Responda os itens da categoria Desempenho Geral considerando a observação do comportamento geral da criança durante toda a sessão.

\begin{tabular}{|c|c|c|c|c|c|}
\hline \multicolumn{6}{|c|}{ DESEMPENHO GERAL } \\
\hline \multirow[t]{3}{*}{ III.1 } & 1. Organização & $\mathrm{F}$ & $\mathrm{O}$ & $\mathrm{N}$ & NO \\
\hline & 2. Planejamento & $\mathrm{F}$ & $\mathrm{O}$ & $\mathrm{N}$ & $\mathrm{NO}$ \\
\hline & 3. Atenção & $\mathrm{F}$ & $\mathrm{O}$ & $\mathrm{N}$ & $\mathrm{NO}$ \\
\hline \multirow[t]{5}{*}{ III. 2} & 1. Auto-julgamento & & & & \\
\hline & 2. Iniciativa & & & & \\
\hline & 3. Decisão & & & & \\
\hline & 4. Ansiedade & & & & \\
\hline & 5. Auto-regulação & & & & \\
\hline
\end{tabular}




\section{Apêndice B}

Roteiro de avaliação do senso de auto-eficácia

NOME:

DATA:

Vou apresentar para você algumas frases e você vai me dizer o quanto elas tem a ver com o seu jeito de ser. Basta me responder SIM quando elas se parecerem e NÃO quando elas forem diferentes do seu jeito.

\begin{tabular}{|l|l|l|}
\hline NA ESCOLA, & SIM & NÃO \\
\hline 01. Eu tenho me saído bem. & & \\
\hline * 02. Eu quero parar de estudar logo. & & \\
\hline 03. Eu consigo ler com facilidade. & & \\
\hline *04. Minha família me considera um aluno fraco. & & \\
\hline 05. Eu consigo copiar com facilidade. & & \\
\hline 06. Minha professora me considera um aluno fraco. & & \\
\hline 07. Eu consigo escrever as palavras que são ditadas. & & \\
\hline 08. Eu demoro mais que os outros para acabar as atividades. & & \\
\hline 09. Eu lembro com facilidade do que aprendi. & & \\
\hline 10. Eu tenho mais dificuldade para a prender que os meus colegas. & & \\
\hline 11. Eu aprendo tão bem quanto os meus colegas. & & \\
\hline 12. Eu esqueço rápido o que aprendi. & & \\
\hline 13. Eu acabo as atividades no mesmo tempo que os colegas. & & \\
\hline 14. Eu tenho dificuldade para escrever as palavras que são ditadas. & & \\
\hline 15. Minha professora me considera um bom aluno. & & \\
\hline 16. Eu tenho dificuldade para fazer cópia. & & \\
\hline * 17. Minha família me considera um bom aluno. & & \\
\hline 18. Eu tenho dificuldade para ler. & & \\
\hline 19. Eu tenho me saído mal. & & \\
\hline *20. Eu quero continuar estudando por muitos anos. & & \\
\hline
\end{tabular}

Estes itens não devem ser precedidos da expressão: "NA ESCOLA". 